\title{
General practitioners and health and safety at work
}

\author{
Some doctors do not believe that risks and regulations apply to them
}

All general practitioners are bound by health and safety law and should be able to show their compliance with such legislation. Surveys have suggested, however, that some doctors either do not know their legal responsibilities or have other reasons for not complying. ' ' This is important given the current trend to hold people legally accountable for their noncompliance. The Health and Safety at Work Act is the umbrella for many regulations, including the Control of Substances Hazardous to Health Regulations (COSHH). Several other European directives will soon become law. These cover the suitability of workplaces, provision of technical and protective equipment, use of video display units, and, crucially for many health care professionals, manual handling of, for example, patients.

The law makes employers responsible for their employees' health and safety at work as far as practicable. Subcontractors, visitors, and patients in waiting rooms are similarly covered by the legislation. Statutory requirements apart, there are common law precedents requiring employers to comply with basic principles of health and safety. Judge Swanwick's test of negligence remains the measure of adequate performance: "The test of negligence is still the conduct of the reasonable and prudent employer, but where there . . . is developing knowledge, we must keep reasonably abreast of it, and must not be slow in applying it: and where he has a greater than average knowledge of the risks, he may, thereby, be obliged to take more than average precautions." ${ }_{+}$ Arguably, doctors and other health care professionals may be considered to have greater than average knowledge of health risks at work.

The principles underlying current health and safety legislation include the assessment and control of risk, health surveillance, and staff training. This applies to all potential health problems related to work, whether chemical, biological, physical, or psychosocial. Despite recommendations by the BMA five years ago that health care workers who are in contact with patients, body fluids, or pathological specimens should be immunised against hepatitis B many doctors either do not believe that they need immunisation or have difficulty in obtaining it." Under the terms of COSSH hepatitis B is a microbiological hazard, and general practitioners should ensure that they and their staff receive proper protection. All staff who are likely to be exposed to body fluids - including those at risk of being bitten or scratched by patients, those taking or handling specimens, and those undertaking or assisting with surgical and dental procedures - should receive a full course of vaccine. Their subsequent immunity should be checked by estimating surface antibody response, which should be greater than $100 \mathrm{mIU} / \mathrm{ml}$. Staff should be trained to avoid accidental inoculation injuries, and a procedure should be prepared for dealing with such events. Immunisation against tuberculosis should be undertaken, according to the British Thoracic Society's code of practice. This recommends Heaf testing of people working with high risk patients or children to see whether they require BCG immunisation.

The use of all chemical substances, including cleaning materials and prescribed medicines, should be assessed. Staff $\frac{0}{3}$

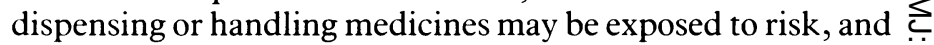
hence an assessment is required. Some drugs, such as $\vec{\Rightarrow}$ antibiotics, carry a risk of occupational asthma and those $\stackrel{\oplus}{\rightarrow}$ handling them must be offered regular respiratory review $\frac{C}{\sigma}$ by questionnaire and spirometry. ${ }^{8}$ Glutaraldehyde is $\frac{\bar{\omega}}{\bar{\omega}}$ another respiratory and skin sensitiser and its use should be avoided.

From the beginning of next year all video display units will have to be assessed: new work stations will have to meet minimum ergonomic standards, and all those who habitually $\vec{\omega}$ use video display units as a substantial part of their work will have the right to eye tests before starting work and peri- $\frac{0}{3}$. odically thereafter. The proposed manual handling regulations are particularly designed to minimise the risks of back ơ injury and require an assessment of any manual handling operation that carries a risk of injury.

Apart from the practical requirements, current legislation $\vec{\circ}$ places an administrative responsibility on all employers. $\$$ Those with five or more employees are required to produce a $ᄋ$ written policy on health and safety and ensure that all staff are $\underline{\omega}$ aware of it. Recognised unions have the right to appoint $\vec{O}$ workplace health and safety representatives. The Reporting of Injuries, Diseases and Dangerous Occurrences Regulations $\underset{\mathbb{\Phi}}{\mathscr{D}}$ (RIDDOR) clearly detail those incidents that must be reported to the Health and Safety Executive-including all $\&$ absences of three or more days resulting from a workplace accident or occupationally related illness.

The Health of the Nation looks to the NHS to set an example to other employers by providing good policies on health promotion in the workplace. "1 These should include measures to address all true occupational health risks but also general issues including smoking in the workplace, alcohol policies, $\overline{3}$ and stress avoidance. Adherence to current and pending legislation will go a long way towards achieving that aim.

Consultant Occupational Physician,

$\mathrm{RA} C O O K E \sum_{\xi}$

Worcester and District Health Authority,

Department of Occupational Health,

Ronkswood Hospital,

Worcester WR5 1JP

Consultant Occupational Physician,

Oxford University Occupational Health Service,

Radcliffe Infirmary,

Oxford OX2 6HE

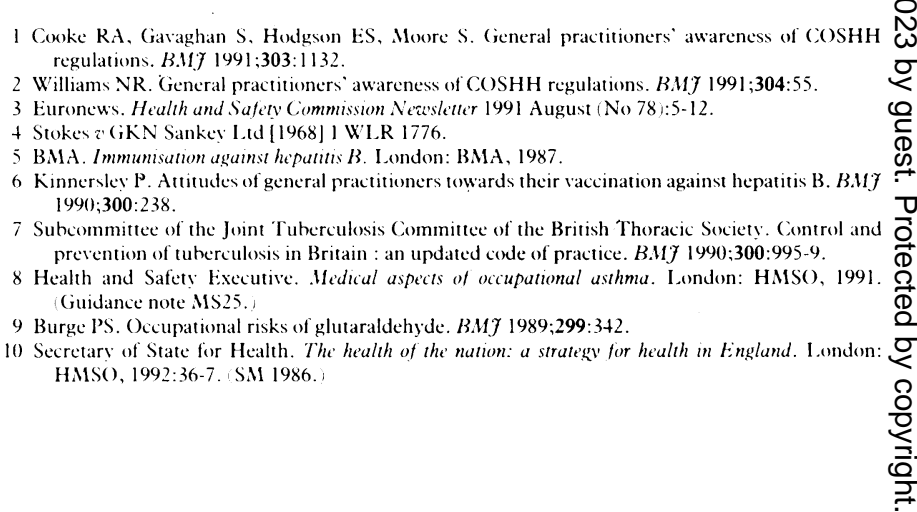

